\title{
Thirty-day mortality and five-year survival in thoracic surgery: "real-world" assessment of outcomes from a single-institution audit
}

\section{(c) (1) ब(9)}

Reprints and permissions: sagepub.co.uk/journalsPermissions.nav DOI: $10.530 \mathrm{l} / \mathrm{tj} .5000709$ journals.sagepub.com/home/tmj (\$)AGE

\author{
Anna Cantarutti ${ }^{1}$, Carlotta Galeone ${ }^{2}$, Giovanni Leuzzi ${ }^{3}$, Elena Bertocchi ${ }^{3}$, Giovanna Pomponi ${ }^{4}$, Martin Langer ${ }^{5}$, \\ Vincenzo Mazzaferro', Gustavo Galmozzi ${ }^{4}$, Giovanni Apolone ${ }^{7}$, Giovanni Corrao ${ }^{1}$, Ugo Pastorino ${ }^{3}$ \\ ${ }^{1}$ Department of Statistics and Quantitative Methods, Division of Biostatistics, Epidemiology and Public Health, University of Milano-Bicocca, \\ Milan - Italy \\ ${ }^{2}$ Department of Clinical Sciences and Community Health, University of Milan, Milan - Italy \\ ${ }^{3}$ Thoracic Surgery Unit, Fondazione IRCCS Istituto Nazionale dei Tumori, Milan - Italy \\ ${ }^{4}$ Medical Directorate, Fondazione IRCCS Istituto Nazionale dei Tumori, Milan - Italy \\ ${ }^{5}$ Anesthesiology Department, Fondazione IRCCS Istituto Nazionale dei Tumori, Milan - Italy \\ ${ }^{6}$ General Surgery Department, Fondazione IRCCS Istituto Nazionale dei Tumori, Milan - Italy \\ ${ }^{7}$ Scientific Director, Fondazione IRCCS Istituto Nazionale dei Tumori, Milan - Italy
}

\begin{abstract}
Background: Accurate measurement of outcomes is essential to monitor the effectiveness of public health policies. In Italy, the Ministry of Health has chosen 30-day mortality after major surgical or medical procedures as the main outcome measure, pooling all pulmonary resections for malignancy in a single category. The present audit evaluated all pulmonary resections performed over a 13-year period in a single institution to assess the immediate (30-day mortality) and long-term (5-year survival) outcomes according to type and stage of disease and extent of surgery.

Methods: We analyzed the results of 4,234 first pulmonary resections performed from 2003 to 2015 for lung cancer (2,636), lung metastases (1,080), other primary cancers (259) and benign diseases (259). The median follow-up of cancer patients was 4.1 years.

Results: Overall 30 -day mortality was $1.1 \%$, being $1.2 \%$ for lung cancer, $0.3 \%$ for lung metastases, $3.5 \%$ for pneumonectomies, $1 \%$ for lobectomies, and $0.5 \%$ for sublobar resections. Among lung cancer patients, $30-$ day mortality was $0.7 \%$ for simple anatomical resections, $2.8 \%$ for complex resections, $0.7 \%$ for stage I, and $1.6 \%$ for higher stages. Overall 5-year survival was $56 \%$ for lung cancer, $49 \%$ for lung metastases, and $53 \%$ for other primary cancers $(p=0.03)$. According to the surgical procedure for lung cancer, 5 -year survival was $60 \%, 55 \%$ and $36 \%$ for lobectomies, segmentectomies and pneumonectomies, respectively $(p<0.0001)$.

Conclusions: For better monitoring of thoracic surgery outcomes in a real-world setting, we suggest evaluating lung cancer separately from other thoracic malignancies, and including 5-year survival rates stratified by resection volume and surgical procedure complexity.
\end{abstract}

Keywords: Lobectomy, Lung cancer, Mortality, Outcome, Survival, Thoracic surgery

\section{Introduction}

In modern medicine, accurate measurements of immediate and long-term outcomes are crucial to monitor the

Accepted: November 16, 2017

Published online: November 21, 2017

Corresponding author:

Ugo Pastorino

Thoracic Surgery Unit

Fondazione IRCCS Istituto Nazionale dei Tumori

Via Venezian 1

20133 Milan, Italy

ugo.pastorino@istitutotumori.mi.it effectiveness of public health policies and to assess the efficiency of intervention, including cost/benefit evaluations, in order to help stakeholders optimize the use of limited financial resources and address research investments. The availability of an independent source of reliable information about the performance of current therapies is fundamental also for increasing patients' awareness of therapeutic strategies.

In the USA, typical examples of public data on medical outcomes are the Surveillance, Epidemiology and End Results (SEER) and Medicare database publications (1). In the last 3 decades, SEER as well as the Society of Thoracic Surgeons (STS) reports on thoracic surgery clearly demonstrated that a higher level of quality, better adherence to guidelines, and a larger number of procedures per year by institution are 


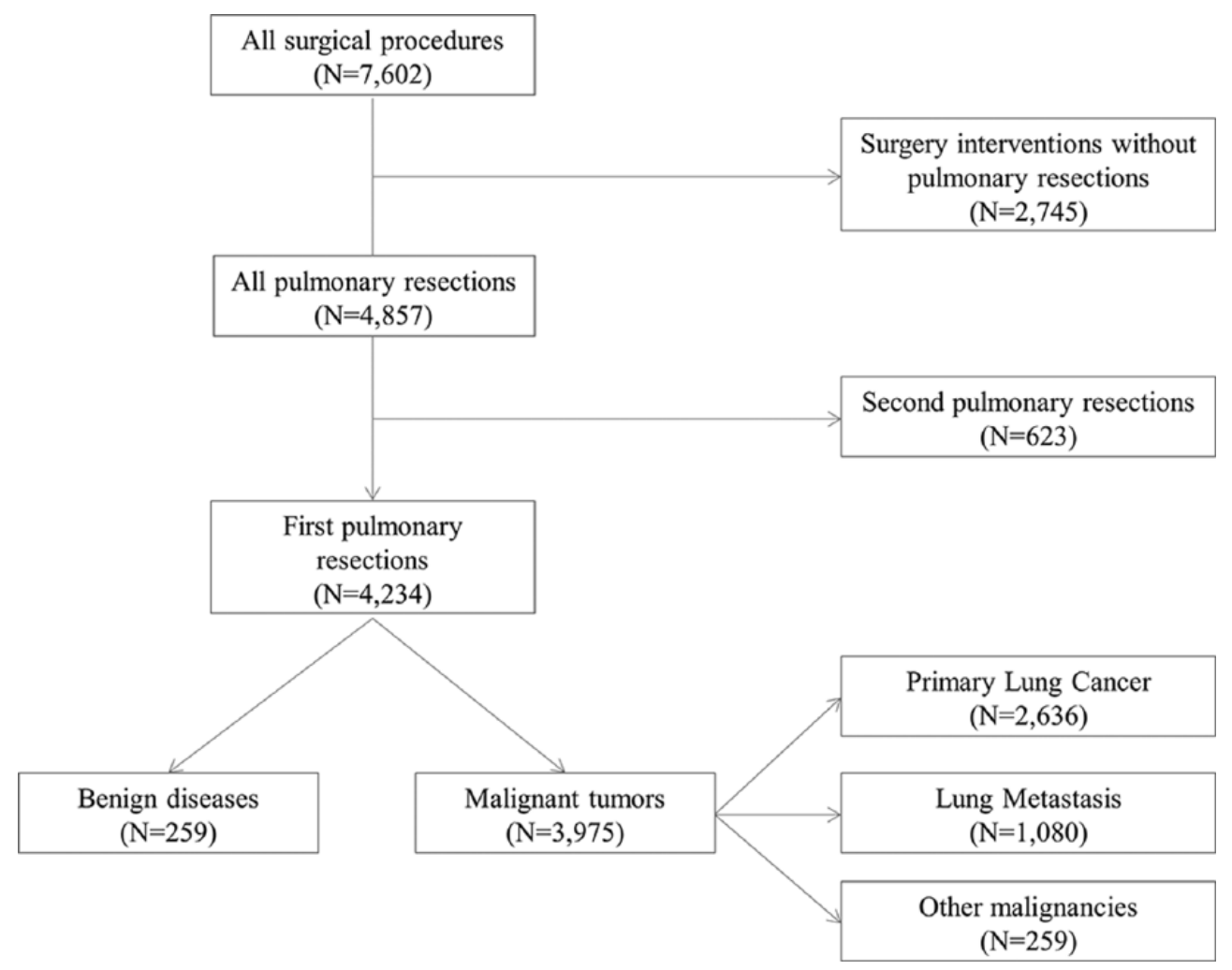

Fig. 1 - Flowchart of the analysis.

associated with lower immediate mortality and better longterm survival (2-7).

Similarly, in Europe, the United Kingdom's second National Thoracic Surgery and Outcomes Report (8) and France's National Cancer Plan based on the Epithor National Database (9) have provided large-scale statistics on thoracic surgery outcomes, including 30-day mortality by procedure. In addition, the European Society of Thoracic Surgeons (ESTS) has designed a database to provide a tool for a fair evaluation of performance across different European thoracic surgery units (10). Furthermore, the ESTS updated risk-adjusted models of morbidity and mortality in order to provide easier instruments of risk assessment for thoracic surgery institutions $(11,12)$.

In Italy, an official agency of the Ministry of Health, the Agenzia Nazionale per i Servizi Sanitari Regionali (AGENAS), provided 30-day mortality statistics after major surgical or medical procedures for each Italian hospital over a period of 6 years (2008-2013) based on administrative data such as discharge summaries and diagnosis-related groups (13). Given the source of the medical data, the AGENAS reports on thoracic surgery combine all pulmonary resections for malignant tumors without distinction of the type of disease (primary lung cancer, lung metastases, other malignancies), extent of resection (whole lung, lobe, lesser volume), and complexity of the procedure (lung only, nodal dissection, extension to adjacent structures, organ replacement or reconstruction). No reports on long-term outcomes are released for the same populations.

The present audit analyzed all pulmonary resections performed at the Istituto Nazionale dei Tumori of Milan (INTM) over a 13-year period with the aim of assessing the immediate and long-term outcomes (30-day mortality and 5-year survival) of surgical procedures according to the type of disease and extent of surgical resection.

\section{Methods}

The current study included all surgical procedures carried out at INTM from January 1, 2003 to December 31, 2015. All resections in pediatric patients (below 18 years of age) were excluded from the cohort. Resections were performed by a variety of operative procedures classified as anatomical resections (pneumonectomy, lobectomy, segmentectomy) or non-anatomical sublobar resections, including wedge and precision resections. In addition, the procedures performed were classified as simple (i.e., not associated with other resections) or complex (associated with resections of adjacent structures, organ replacement or reconstruction). For patients with primary lung cancer, simple anatomical resections always included systematic dissection of multiple hilar and mediastinal lymph-node stations. All anatomical resections for primary lung cancer were staged according to the $7^{\text {th }}$ edition of the International Association for the Study of Lung Cancer (IASLC) staging system (14). All information relating to the period of hospitalization were validated through the medical record register, particularly for patients who died during hospitalization.

Of the 7,602 surgical procedures recorded we included only the first pulmonary resection performed in each individual, corresponding to a total of 4,234 patients (Fig. 1). Benign diseases were analyzed separately (Supplementary Table S1 Thirty-day mortality and postoperative hospital stay in patients with benign diseases. Available online at www.tumorijournal. com). Two outcomes (proportion of patients who experienced 
30-day mortality, and Kaplan-Meier estimate of 5-year survival) were calculated.

Outcomes were stratified according to (i) cancer type (primary lung cancer, lung metastases, and other malignancies), (ii) resection volume (pneumonectomy, lobectomy, segmentectomy, and wedge or precision sublobar resections), (iii) complexity of resection (simple or complex), and (iv) pathological TNM stage.

Additionally, we calculated the $95 \%$ confidence intervals $(\mathrm{Cl})$ of 30-day mortality and performed adjusted Cox proportional hazard regression models to estimate the hazard ratios for all variables of interest just described. Model adjustments were made in all patients for age ( $\leq 64$ years) and sex, and in the lung cancer subgroup for age, sex, anatomical resection and pathological TNM stage (Supplementary Table S2 - Adjusted hazard ratios and 95\% confidence intervals in lung cancer patients according to pathological stage and anatomical resections. Available online at www. tumorijournal.com).

The INTM Medical Directorate checked the in-hospital deaths. All other deaths were retrieved through record linkage with the vital status National Registry Office database, which is regularly updated within 6 months of death and is the same as the AGENAS mortality data. Six patients $(0.1 \%)$ were lost to follow-up. The closing date of follow-up for alive patients was April 30, 2016, and the median follow-up of cancer patients was 4.1 years.

All analyses were performed using the Statistical Analysis System software (version 9.4; SAS Institute, Cary, NC, USA). Statistical significance was set at the 0.05 level.

\section{Results}

Of the 7,602 thoracic surgical procedures recorded at INTM from January 2003 to December 2015, 4,857 (64\%) were pulmonary resections and $4,234(87 \%)$ represented the first surgical procedure (Fig. 1). The remaining 623 subsequent lung resections were not included in the analysis.

The patients' characteristics are illustrated in Table I. Out of 4,234 first pulmonary resections, 259 (6.1\%) were performed for benign diseases. Among the 3,975 patients (93.9\%) with malignant tumors, 2,636 $(66.3 \%)$ had primary lung cancer, 1,080 (27.2\%) lung metastases, and 259 (6.5\%) other primary malignancies. The most common resection performed was lobectomy (51\%), followed by wedge or precision sublobar resections (30\%), anatomical segmentectomies $(9.8 \%)$, and pneumonectomies $(9.2 \%)$.

\section{Thirty-day mortality}

Table II shows the 30-day mortality in patients with malignant tumors according to cancer type and resection volume. Patients with other primary malignancies (thymoma, sarcoma or germ-cell tumors) had the highest mortality (3.1\%) while those with lung metastases had the lowest mortality $(0.3 \%)$, independent of the resection volume. Pneumonectomy was associated with a significantly higher mortality rate $(3.5 \%)$ than lobectomy $(1 \%)$, segmentectomy $(0.5 \%)$ or wedge/precision resection $(0.5 \%)$, regardless of the type of disease. In lung cancer patients, 30-day mortality (1.2\% overall) varied according to the resection volume and complexity of the surgical procedure (Tab. II), being higher for pneumonectomies $(3.1 \%)$ versus lobectomies $(1.1 \%)$ or sublobar resections $(0.6 \%$ combining segments and wedges), with a major impact of complex resections compared to simple procedures $(2.8 \%$ vs. $0.7 \%$ overall), which was evident for pneumonectomy ( $3.8 \%$ vs. $2.0 \%$ ), lobectomy $(2.5 \%$ vs. $0.7 \%$ ) and sublobar resections ( $2.6 \%$ vs. $0.4 \%)$. Table II also shows the 30-day mortality according to pathological tumor stage in 2,376 lung cancer patients undergoing anatomical resections. Stage I lung cancer had a significantly lower mortality than higher stages ( $0.7 \%$ vs. $1.6 \%)$, and the effect was evident in the largest group of 1,845 lobectomies $(0.7 \%$ vs. $1.3 \%)$ as well as in pneumonectomies (0\% vs. $3.6 \%$ ).

Compared to cancer patients, postoperative mortality was lower but not absent $(0.4 \%)$ in lung resections for benign disease (Supplementary Table S1). The $95 \% \mathrm{Cls}$ of 30 -day mortality were $0.8-1.4$ overall, $0.8-1.7$ for primary lung cancer, $0.7-1.6$ for lobectomies, and 2.1-5.9 for pneumonectomies.

TABLE I - Selected cohort characteristics in patients with malignant tumors according to cancer type

\begin{tabular}{|c|c|c|c|c|c|c|c|c|}
\hline \multirow{2}{*}{ Sex } & \multicolumn{2}{|c|}{$\begin{array}{c}\text { Overall } \\
(\mathbf{N}=3,975)\end{array}$} & \multicolumn{2}{|c|}{$\begin{array}{l}\text { Lung cancer } \\
\qquad(N=2,636)\end{array}$} & \multicolumn{2}{|c|}{$\begin{array}{l}\text { Metastasis } \\
(N=1,080)\end{array}$} & \multicolumn{2}{|c|}{$\begin{array}{c}\text { Other } \\
(\mathrm{N}=\mathbf{2 5 9})\end{array}$} \\
\hline & & & & & & & & \\
\hline Female, n (\%) & 1,464 & $(36.8)$ & 888 & $(33.7)$ & 478 & $(44.3)$ & 98 & $(37.8)$ \\
\hline Male, n (\%) & 2,511 & $(63.2)$ & 1,748 & $(66.3)$ & 602 & $(55.7)$ & 161 & $(62.2)$ \\
\hline Age, mean (SD), years & 61.7 & $(13.0)$ & 64.9 & $(10.6)$ & 56.0 & $(14.5)$ & 52.9 & $(15.7)$ \\
\hline Body mass index, mean $(S D)^{\ddagger}$ & 25.6 & (3.9) & 25.61 & (3.9) & 26.03 & $(2.8)$ & 25.68 & $(0.3)$ \\
\hline \multicolumn{9}{|l|}{ Resection volume } \\
\hline Pneunomectomy, n (\%) & 367 & $(9.2)$ & 261 & (9.9) & 11 & $(1.0)$ & 95 & $(36.7)$ \\
\hline Lobectmy, n (\%) & 2,027 & $(50.9)$ & 1,845 & $(69.9)$ & 141 & $(13.1)$ & 41 & $(15.8)$ \\
\hline Segmentectomy, n (\%) & 390 & $(9.8)$ & 270 & $(10.2)$ & 113 & $(10.5)$ & 7 & $(2.7)$ \\
\hline Wedge/precision, n (\%) & 1,191 & (29.9) & 260 & (9.9) & 815 & $(75.5)$ & 116 & $(44.8)$ \\
\hline
\end{tabular}

${ }^{\ddagger}$ Missing data are hidden. 
In summary, the highest mortality was observed in patients undergoing pneumonectomy, but the type of malignancy, the complexity of resection, and the pathological stage of lung cancer played a significant role. In particular, complex resections showed a higher mortality rate than simple resections regardless of the resected lung volume.

Overall, the median postoperative hospital stay was 6 days (Tab. III), being longer for primary tumors other than lung cancer ( 9 days) and pneumonectomies ( 8 days), and shorter for lung metastases and wedge and precision resections (5 days).

\section{Overall survival}

Six patients $(0.1 \%)$ were excluded from the survival analysis due to missing follow-up. Overall, 16,309.62 person-years were accumulated by patients with malignant tumors and 1,870 deaths occurred during the follow-up period, with a mortality rate for all causes of $11.5 / 100$ person-years. The 5-year survival according to cancer type showed a significant difference between patients with lung cancer, other primary malignancies, and lung metastases, with a cumulative survival of $56 \%, 53 \%$, and $49 \%$, respectively ( $p=0.03$; Fig. 2 ). Compared with primary lung cancer patients, those with lung metastases and other malignancies had an adjusted hazard ratio (HR) of 1.21 (95\% Cl 1.08-1.36) and 1.26 (1.03-1.54), respectively (Tab. IV). When the analysis was restricted to lung cancer patients and anatomical resections, 5-year survival was lower in patients undergoing pneumonectomy compared with lobectomy and segmentectomy; the difference was statistically significant ( $36 \%$ vs. $60 \%$ and $55 \%$, p<0.0001; Fig. 3). Patients who underwent pneumonectomies had worse survival compared with all other resection types (HR 1.91; 95\% $\mathrm{Cl}$ 1.49-2.46; Tab. IV). Also, a significant outcome stratification was evidenced by combining complexity of the surgical procedure and lung resection volume $(p<0.0001$; Fig. 4), with an HR of 1.96 for simple pneumonectomies and 2.59 for complex ones (Tab. IV).

TABLE III - Postoperative hospital stay in malignant tumors according to resection volume and type of disease

\begin{tabular}{|c|c|c|c|c|c|}
\hline & & LC & META & Other & Total \\
\hline \multirow[t]{2}{*}{ Pneumonectomy } & Median (IQR) & $7(4)$ & $7(5)$ & $12(10)$ & $8(6)$ \\
\hline & $N$ & 261 & 11 & 95 & 367 \\
\hline \multirow[t]{2}{*}{ Lobectomy } & Median (IQR) & $6(4)$ & $6(2)$ & $8(6)$ & $6(4)$ \\
\hline & $N$ & 1,845 & 141 & 41 & 2,027 \\
\hline \multirow[t]{2}{*}{ Segmentectomy } & Median (IQR) & $6(3)$ & $5(1)$ & $5(7)$ & $6(2)$ \\
\hline & $N$ & 270 & 113 & 7 & 390 \\
\hline \multirow[t]{2}{*}{ Wedge/precision } & Median (IQR) & $5(3)$ & $5(2)$ & $7(6)$ & $5(2)$ \\
\hline & $N$ & 260 & 815 & 116 & 1,191 \\
\hline \multirow[t]{2}{*}{ Total } & Median (IQR) & $6(3)$ & $5(2)$ & $9(7)$ & $6(3)$ \\
\hline & $\mathrm{N}$ & 2,636 & 1,080 & 259 & 3,975 \\
\hline
\end{tabular}

$I Q R=$ interquartile range; $L C=$ lung cancer; $M E T A=$ lung metastasis; $N=$ number. 


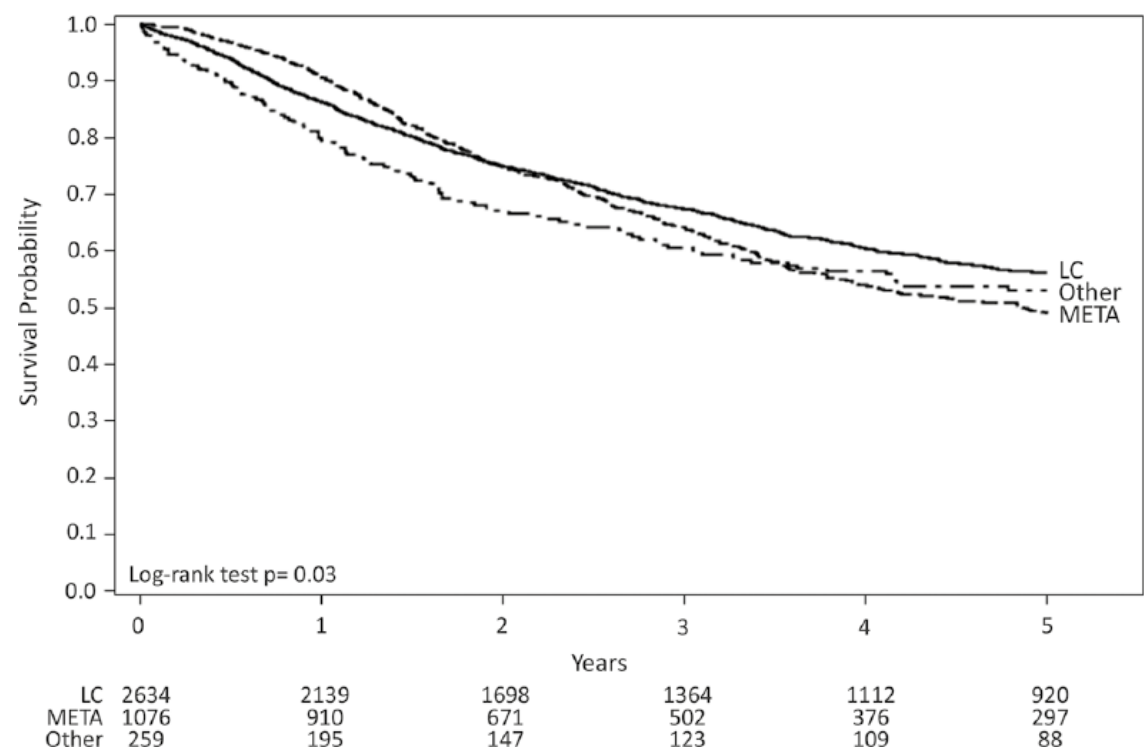

Fig. 2 - Five-year survival according to cancer type: primary lung cancer (LC), lung metastases (META), and other malignancies (Other).
TABLE IV - Adjusted hazard ratios and 95\% confidence intervals according to different settings

\begin{tabular}{|c|c|c|}
\hline & $\mathrm{HR}^{*}$ & $(95 \% \mathrm{Cl})$ \\
\hline \multicolumn{3}{|c|}{$\begin{array}{l}\mathrm{HR}(95 \% \mathrm{Cl}) \text { according to cancer type: primary } \\
\text { lung cancer, lung metastases and other } \\
\text { malignancies }\end{array}$} \\
\hline LC & 1.00 & (reference) \\
\hline META & 1.21 & $(1.08-1.36)$ \\
\hline Other & 1.26 & $(1.03-1.54)$ \\
\hline \multicolumn{3}{|c|}{$\begin{array}{l}\mathrm{HR}(95 \% \mathrm{Cl}) \text { in } \mathrm{LC} \text { patients according to ana- } \\
\text { tomical resections: lobectomy, segmentec- } \\
\text { tomy and pneumonectomy }\end{array}$} \\
\hline Lobe. & 1.00 & (reference) \\
\hline Segment. & 1.08 & $(0.88-1.33)$ \\
\hline Pneumo. & 2.07 & $(1.73-2.47)$ \\
\hline \multicolumn{3}{|c|}{$\begin{array}{l}\mathrm{HR}(95 \% \mathrm{Cl}) \text { in } \mathrm{LC} \text { patients according to anatomi- } \\
\text { cal resections and complexity of procedure }\end{array}$} \\
\hline Lobe./Segment. SIMPLE & 1.00 & (reference) \\
\hline Lobe./Segment. COMPLEX & 1.67 & $(1.42-1.96)$ \\
\hline Pneumo. SIMPLE & 1.96 & $(1.48-2.59)$ \\
\hline Pneumo. COMPLEX & 2.59 & $(2.08-3.23)$ \\
\hline \multicolumn{3}{|c|}{$\begin{array}{l}\mathrm{HR}(95 \% \mathrm{Cl}) \text { in } \mathrm{LC} \text { patients with simple ana- } \\
\text { tomical resections according to pathological } \\
\text { stage }\end{array}$} \\
\hline Stage I & 1.00 & (reference) \\
\hline Stage II & 2.11 & $(1.75-2.55)$ \\
\hline Stage III-IV & 3.57 & $(3.04-4.18)$ \\
\hline \multicolumn{3}{|c|}{$\begin{array}{l}\mathrm{HR}(95 \% \mathrm{Cl}) \text { in LC patients with complex anatom- } \\
\text { ical resections according to pathological stage }\end{array}$} \\
\hline Stage I & 1.00 & (reference) \\
\hline Stage II & 2.10 & $(1.40-3.15)$ \\
\hline Stage III-IV & 3.43 & $(2.35-4.99)$ \\
\hline
\end{tabular}

$\mathrm{Cl}=$ confidence interval; $\mathrm{HR}=$ hazard ratio; $\mathrm{LC}=$ lung cancer; Lobe. $=$ lobec tomy; Pneumo. = pneumonectomy; Segment. = segmentectomy.

*Adjusted for age and sex.
Finally, 5-year survival by pathological tumor stage confirmed the expected outcome according to the new TNM staging system: $73 \%$ for stage I, $55 \%$ for stage II, and $36 \%$ for stage III-IV ( $p<0.0001$; Fig. 5), and patients with pathological stage II or III had an increased risk of long-term mortality compared to stage I patients (HR 2.1,95\% Cl 1.40-3.15 and HR 3.4, $95 \% \mathrm{Cl}$ 2.35-4.99, respectively; Tab. IV). Interestingly, a similar impact of PTNM on 5-year survival was observed when restricting the analysis to the 580 patients treated by complex resections only ( $72 \%$ vs. $49 \%$ and $29 \%$, respectively, $p<0.000$; HR 2.1 and 3.43; Tab. IV and Supplementary Fig. S1- Five-year lung cancer survival of anatomical resections according to pathological stage in patients with complex resections. Available online at www.tumorijournal.com). The independent predictive value of anatomical resection volume and PTNM was maintained after multiple adjustment for age, sex, anatomical resection and pTNM, with an HR of 1.54 for pneumonectomy and 3.37 for stage III-IV (Supplementary Table S2).

\section{Discussion}

Real-world medicine, as opposed to evidence-based medicine generated by prospective randomized trials, mainly refers to the retrospective evaluation of large unselected series of patients, including all those treated in one or more hospitals, whose clinical results cannot be affected by the selection biases that are typical in the process of trial accrual. Real-world evidence provides a complementary overview (or confirmation) of the results that can be achieved in the large majority of "real" patients routinely treated in clinical practice, including elderly and frail patients with comorbidities, who are usually excluded from randomized trials (15). In the setting of thoracic surgery, large-scale multicenter databases have been employed both in the USA (SEER, STS) and Europe (ESTS) in an effort to provide reliable short- and long-term outcome results and easy-to-use quality indicators of institutional performance (1-12).

On the other hand, major single institutions can use the audit instrument to analyze their own surgical databases and 

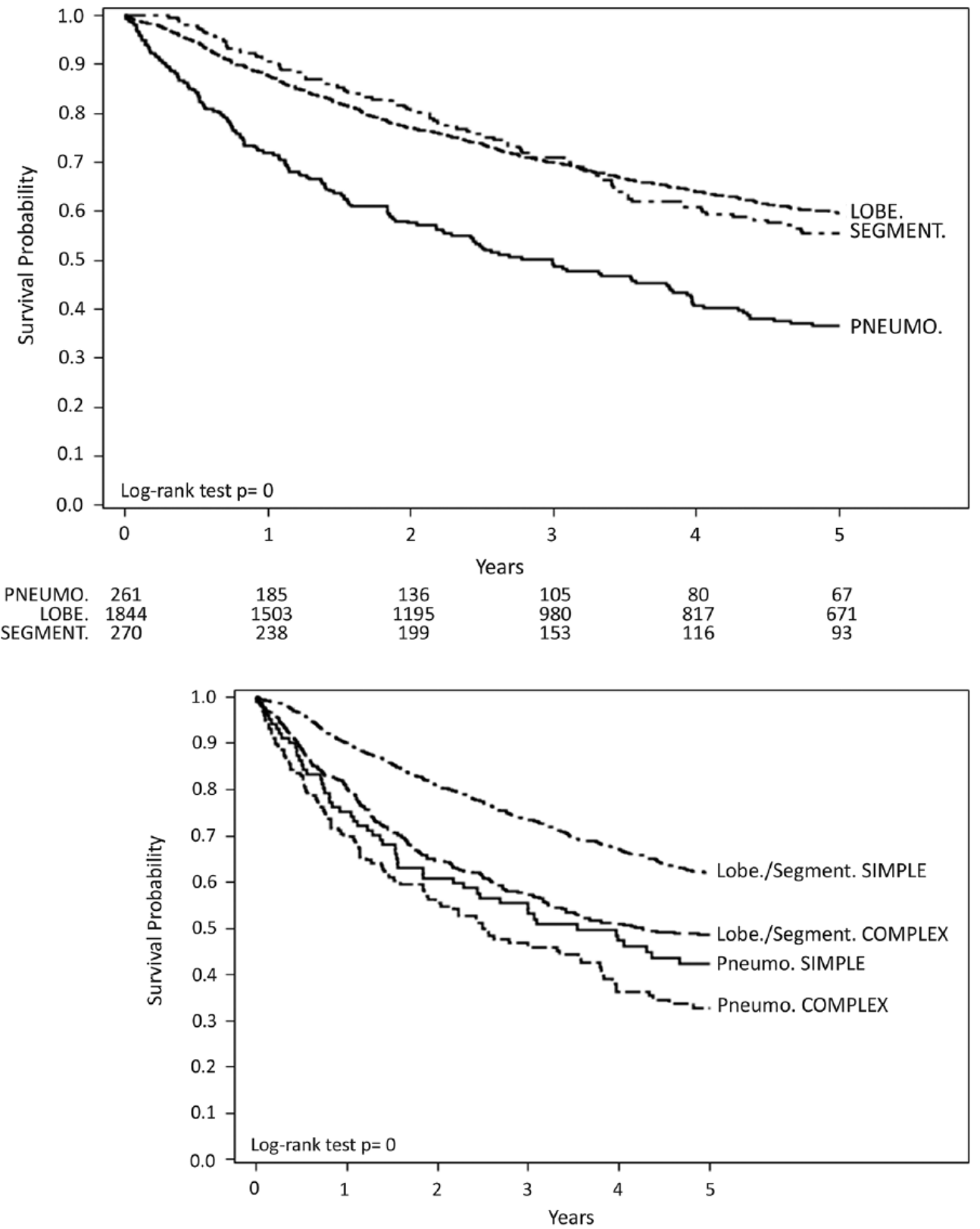

Fig. 3 - Five-year lung cancer survival according to anatomical resections: pneumonectomy (PNEUMO), segmentectomy (SEGMENT), and lobectomy (LOBE).
Fig. 4 - Five-year lung cancer survival according to anatomical resections and procedure complexity.

\begin{tabular}{|c|c|c|c|c|c|c|}
\hline $\begin{array}{r}\text { Pneumo. SIMPLE } \\
\text { Pneumo. COMPLEX } \\
\text { Lobe./Segment. SIMPLE } \\
\text { Lobe./Segment. COMPLEX }\end{array}$ & $\begin{array}{c}102 \\
159 \\
1683 \\
431\end{array}$ & $\begin{array}{c}74 \\
111 \\
1407 \\
334\end{array}$ & $\begin{array}{c}56 \\
80 \\
1138 \\
256\end{array}$ & $\begin{array}{l}47 \\
58 \\
929 \\
204\end{array}$ & $\begin{array}{c}39 \\
41 \\
762 \\
171\end{array}$ & $\begin{array}{c}31 \\
36 \\
612 \\
152\end{array}$ \\
\hline
\end{tabular}

provide updated outcome indicators to be shared with patients. In order to avoid retrospective clinical selection and potential biases, an institutional audit should identify all treated patients within a given period through independent medical and administrative databases, regardless of their private or public status, with formal checking by institutional authorities. For the present audit, we merged all the existing databases at INTM, from the ICT, clinical administration, pathology, surgery, and anesthesiology departments, to retrieve each and every patient, including those admitted to medical or private wards and intensive care units.

Even though 30-day mortality is only one measure of the overall health-care quality (11), our outcome analysis was based on all-cause mortality, which represents the most solid endpoint for this type of institutional audit. In contrast with the majority of surgical series, where follow-up was obtained through the outpatient clinic or telephone calls to patients' families, we used systematic record linkage with the vital status National Registry Office database, which is regularly updated within 6 months of death, and in our cohort guaranteed a $99 \%$ long-term follow-up. Other relevant endpoints such as tumor relapse, new primary cancer, adjuvant or salvage treatment are very difficult to accurately identify in a large-scale surgical audit and are beyond the scope of the present exercise.

We are aware that the adjustment for age and sex, anatomical resection and PTNM stage but not other important 


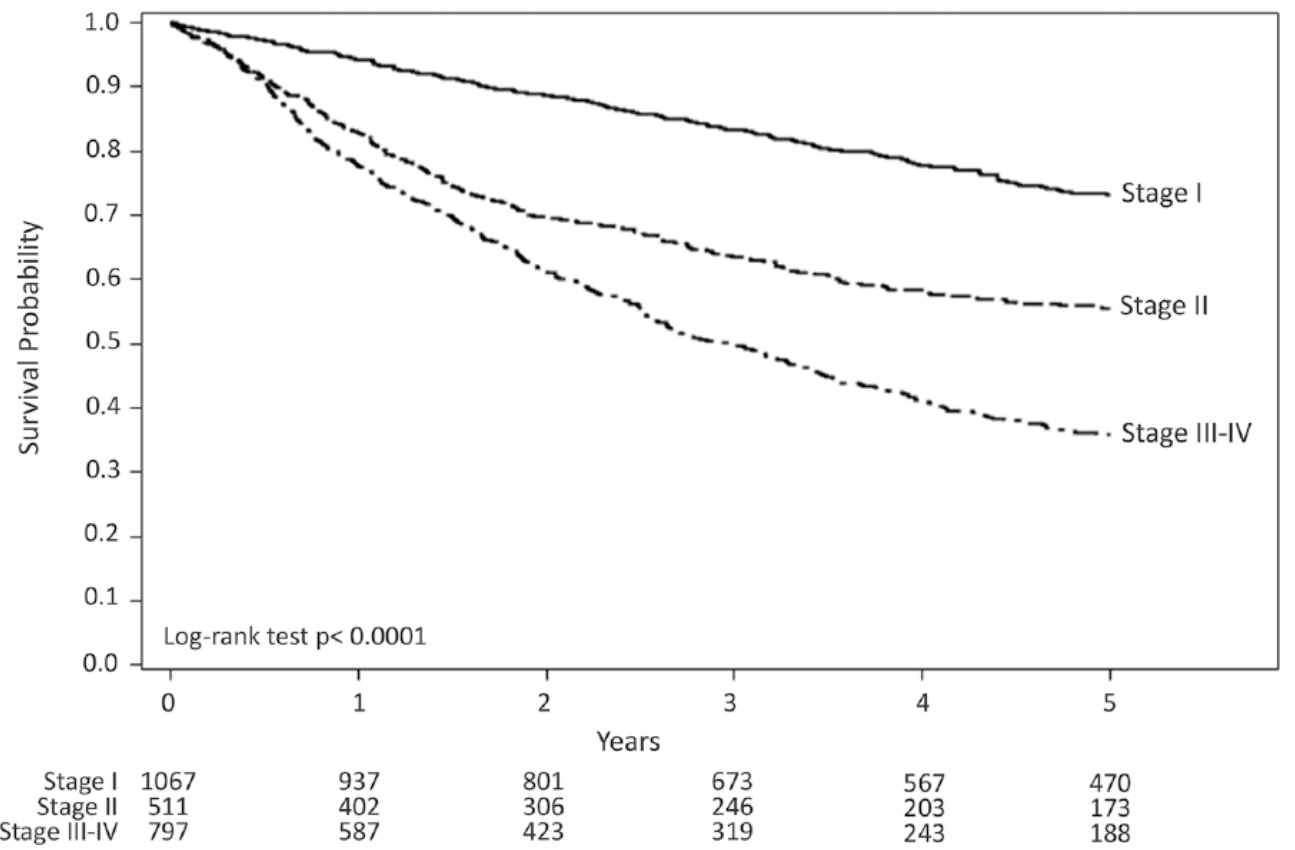

Fig. 5 - Five-year lung cancer survival for anatomical resections according to pathological stage.

TABLE V - Thirty-day mortality (\%) from large-scale and hospital series of lung cancer resections

\begin{tabular}{lccccc}
\hline First author (ref.) & Years & Number & Overall & Lobectomy & Pneumonectomy \\
\hline Birkmeyer (3) & $1994-1999$ & 16,785 & 7.6 & 6.4 & 17 \\
Pastorino (19) & $1998-2002$ & 1,418 & 1.8 & 2.7 & - \\
Kozower (16) & $2002-2008$ & 18,800 & 2.2 & 6.1 & - \\
Falcoz (9) & $2005-2010$ & 19,556 & 7.4 & - & - \\
Hu (1) & $2006-2010$ & 11,787 & 3.7 & 2.3 & 6.8 \\
Brunelli (18) & $2007-2015$ & 47,960 & 2.7 & 1.1 & 3.1 \\
Present series & $2003-2015$ & 2,636 & 1.2 & &
\end{tabular}

risk modifiers such as concurrent chronic diseases, cardiopulmonary function and medical comorbidity is a limitation of the present audit $(16,17)$, but these data were not available for a significant proportion of patients. It is our intention, however, to provide in the near future additional outcome analyses of the lung cancer cohort, adjusted for chronic obstructive pulmonary disease, prior cardiac morbidity, pulmonary function, and other medical comorbidities.

In general terms, our results confirm that overall 30-day mortality in thoracic surgery has declined in the last 20 years (Tab. V), but there are still significant variations among different series, even in the last decade. In fact, the $1.3 \%$ value observed in our series for major lung cancer resections (lobectomy or pneumonectomy) is similar to the most recent STS outcomes $(0.9 \%-1.8 \%)(6)$ but much lower than the $2.7 \%$ reported by ESTS (18), the $3.7 \%$ of SEER-Medicare (1), and the $7.4 \%$ of Epithor (9). Even though the size difference between single-institution and population-based series is substantial, our $95 \% \mathrm{Cls}$ of 30 -day mortality confirm the favorable trend (Tab. IV).

In the specific Italian scenario, our overall 30-day mortality for malignant disease $(1.1 \%)$ is in line with the average national value published by AGENAS, showing a gradual decline from $1.8 \%$ in 2010 to $1.2 \%$ in 2015 (13). Nonetheless, the present audit clearly demonstrates that mortality is influenced by a number of major clinical factors including type and stage of disease, resection volume, and complexity of the surgical procedure. In this context, our distinction between complex and simple procedures follows the new ESTS proposal for 30-day mortality analysis, which separates mortality after extended and non-extended procedures $(5.3 \%$ and $2.5 \%$, respectively) (18). The independent impact of lung cancer stage on 30-day mortality confirms a prior observation from 2 hospital series (19) and is in keeping with the French national report, showing an odds ratio of 1.3 for stage II and 2.2 for stage III compared with stage I in multivariate analysis (9).

All the above-mentioned factors can be very useful to predict the risk of postoperative death in individual patients but also to stratify clinical series, whose composition can be very different according to referral status in highly specialized centers versus general hospitals. Consequently, interinstitutional comparisons are meaningful only within a predefined homogeneous group of patients (e.g., those undergoing lobectomy 
for primary lung cancer) and/or after proper adjustment for major determinants of the surgical risk (20). The lack of homogeneous classification or adequate adjustment of clinical series is a possible explanation for the apparent discrepancy between worldwide outcome figures and the AGENAS reports, where small centers with very limited numbers of lung resections per year often show mortality rates below the average national value (21).

A further element to be considered as a marker of potentially unnecessary surgery is the frequency of lung resections for benign diseases. In the present series, only $6 \%$ of patients underwent pulmonary resection without a final pathological diagnosis of malignancy. This value is well below the $10 \%$ threshold considered adequate by international thoracic surgery guidelines (22).

In our view, the most valuable piece of information provided by this audit is the correlation between short-term mortality and long-term outcome of surgical resections for thoracic malignancies. In fact, the overall 5-year survival of $56 \%$ for 2,634 lung cancer patients and $53 \%$ for 259 subjects with other primary cancers represent solid proof of long-term effectiveness. Even more remarkably, 1,076 patients with resected lung metastases showed a 49\% 5-year survival, with a substantial prospect of permanent cure in carefully selected metastatic disease, and very low $(0.3 \%)$ postoperative mortality. The surgical benefit ratio appears reasonable also for extended operations such as complex pneumonectomies in lung cancer patients, where a 30 -day mortality of $3.8 \%$ was compensated by an acceptable 9 -fold 5 -year survival (35\%).

Finally, the observed long-term outcome provides reassuring information on the appropriateness of patient selection as well as on the quality of surgical management. In fact, the stage-related 5-year survival among lung cancer patients was fully consistent with the new international TNM figures (14). In particular, the 36\% 5-year survival shown in 798 stage III-IV patients matches very well with a 1.6\% 30-day mortality, and is above the average life expectancy with definitive chemoradiotherapy, and the most recent population-based SEER report showing a $29 \% 5$-year survival rate for stage II-III disease and $5 \%$ for stage IV (23). This audit thereby confirms the validity of the surgical choice in terms of the balance between early mortality and late survival also in advanced-stage lung cancer.

Non-anatomical sublobar resections or lobectomies without hilar and mediastinal lymph node dissection may be linked to a $0.5 \%$ to $1 \%$ reduction of 30 -day mortality, but the SEER reports clearly demonstrate that the ultimate cost of suboptimal surgery for primary lung cancer is a $10 \%$ to $20 \%$ reduction of 5 -year survival $(2,4)$. In the present series, lobectomy plus systematic nodal dissection yielded a $60 \%$ 5 -year survival rate in lung cancer patients (all stages) at the cost of a 30 -day mortality of $1.1 \%$. For these reasons, periodic statistics provided by national health authorities such as AGENAS should include 30-day mortality of homogeneous groups of patients (e.g., lobectomy for primary lung cancer) as well as the corresponding 5 -year survival.

\section{Conclusion}

In conclusion, 30-day mortality in thoracic surgery is significantly associated with the type and stage of disease, lung resection volume, and complexity of surgical procedure. Postoperative mortality and 5-year survival are 2 complementary measures of performance, and for better monitoring of thoracic surgery outcomes in a real-world setting we suggest evaluating lung cancer separately from other thoracic malignancies, and including 5 -year survival rates stratified by resection volume and surgical procedure complexity.

\section{Acknowledgments}

The authors thank Dr. Paolo Girotti, Dr. Leonardo Duranti, Dr. Luca Tavecchio, Dr. Lara Girelli, Dr. Stefano Sestini, Paolo Scanagatta and Dr. Luigi Rolli of the Thoracic Surgery Unit for retrieval of patient management information, and Claudio Jacomelli for data management.

\section{Disclosures}

Financial support: The Milan Thoracic Research Program was supported by a research grant from the Italian Ministry of Health (RF 2004 and 2010), Cariplo Foundation (2003), and Lombardy Region (2006/1688).

Conflict of interest: The authors have no conflicts of interest related to this article.

\section{References}

1. Hu Y, McMurry TL, Wells KM, Isbell JM, Stukenborg GJ, Kozower BD. Postoperative mortality is an inadequate quality indicator for lung cancer resection. Ann Thorac Surg. 2014;97(3):973-979.

2. Bach PB, Cramer LD, Schrag D, Downey RJ, Gelfand SE, Begg CB. The influence of hospital volume on survival after resection for lung cancer. N Engl J Med. 2001;345(3):181-188.

3. Birkmeyer JD, Siewers AE, Finlayson EVA, et al. Hospital volume and surgical mortality in the United States. N Engl J Med. 2002;346(15):1128-1137.

4. Varlotto JM, Recht A, Nikolov M, Flickinger JC, Decamp MM. Extent of lymphadenectomy and outcome for patients with stage I nonsmall cell lung cancer. Cancer. 2009;115(4):851-858.

5. Raymond DP, Seder CW, Wright CD, et al. Predictors of major morbidity or mortality after resection for esophageal cancer: a Society of Thoracic Surgeons General Thoracic Surgery Database risk adjustment model. Ann Thorac Surg. 2016;102(1):207-214.

6. Seder CW, Raymond DP, Wright CD, et al. The Society of Thoracic Surgeons General Thoracic Surgery Database 2017 update on outcomes and quality. Ann Thorac Surg. 2017;103(5):1378-1383.

7. The Society of Thoracic Surgeons. General Thoracic Surgery Database public reporting. Available at http://publicreporting.sts. org/gtsd. Accessed November 17, 2017).

8. The Society for Cardiothoracic Surgery in Great Britain \& Ireland. Second National Thoracic Surgery Activity \& Outcomes Report 2011. Available at http://www.scts.org/_userfiles/ resources/634558869917493937_Thoracic_2011_FINAL.pdf. Accessed December 29, 2012.

9. Falcoz PE, Puyraveau M, Rivera C, et al. Epithor Group. The impact of hospital and surgeon volume on the 30-day mortality of lung cancer surgery: a nation-based reappraisal. J Thorac Cardiovasc Surg. 2014;148(3):841-848.

10. Brunelli A, Rocco G. Clinical and nonclinical indicators of performance in thoracic surgery. Thorac Surg Clin. 2007;17(3): 369-377.

11. Brunelli A, Varela G, Van Schil P, et al. ESTS Audit and Clinical Excellence Committee. Multicentric analysis of performance after major lung resections by using the European Society Objective Score (ESOS). Eur J Cardiothorac Surg. 2008;33(2): 284-288. 
12. Brunelli A, Berrisford RG, Rocco G, Varela G; European Society of Thoracic Surgeons Database Committee. The European Thoracic Database project: composite performance score to measure quality of care after major lung resection. Eur J Cardiothorac Surg. 2009;35(5):769-774.

13. Ministero della Salute - Agenzia Nazionale per i Servizi Sanitari Regionali, Programma Ente Nazionale Esiti PNE 2016. Available at http://95.110.213.190/PNEedizione16_p/risultati/tipo1/intr_ struasl1_HC.php?ind=63\&tipo=1\&area $=2$. Accessed November $16,2017$.

14. Goldstraw P, Chansky K, Crowley J, et al. The IASLC Lung Cancer Staging Project: proposals for revision of the TNM stage groupings in the forthcoming (eighth) edition of the TNM classification for lung cancer. J Thorac Oncol. 2016;11(1):39-51.

15. Booth CM, Tannock IF. Randomised controlled trials and population-based observational research: partners in the evolution of medical evidence. Br J Cancer. 2014;110(3):551-555.

16. Kozower BD, Sheng S, O'Brien SM, et al. STS database risk models: predictors of mortality and major morbidity for lung cancer resection. Ann Thorac Surg. 2010;90(3):875-883.

17. Kozower BD, O'Brien SM, Kosinski AS, et al. The Society of Thoracic Surgeons composite score for rating program performance for lobectomy for lung cancer. Ann Thorac Surg. 2016;101(4):1379-1387.
18. Brunelli A, Salati M, Rocco G, et al. ESTS Database Committee. European risk models for morbidity (EuroLung1) and mortality (EuroLung2) to predict outcome following anatomic lung resections: an analysis from the European Society of Thoracic Surgeons database. Eur J Cardiothorac Surg. 2017;51(3): 490-497.

19. Pastorino $U$, Borasio $P$, Francese $M$, et al. Lung cancer stage is an independent risk factor for surgical mortality. Tumori. 2008;94(3):362-369.

20. Fernandez FG, Falcoz PE, Kozower BD, Salati M, Wright CD, Brunelli A. The Society of Thoracic Surgeons and the European Society of Thoracic Surgeons general thoracic surgery databases: joint standardization of variable definitions and terminology. Ann Thorac Surg. 2015;99(1):368-376.

21. Livingston $\mathrm{EH}, \mathrm{Cao} J$. Procedure volume as a predictor of surgical outcomes. JAMA. 2010;304(1):95-97.

22. Smith MA, Battafarano RJ, Meyers BF, Zoole JB, Cooper JD, Patterson GA. Prevalence of benign disease in patients undergoing resection for suspected lung cancer. Ann Thorac Surg. 2006;81(5):1824-1829.

23. National Cancer Institute. Surveillance, Epidemiology, and End Results Program. Cancer Stat Facts: lung and bronchus cancer. https://seer.cancer.gov/statfacts/html/lungb.html. Accessed July 31, 2017. 